\title{
EMT influences the expression of CK19 in pleural effusion-derived lung cancer cells and their invasion and metastasis
}

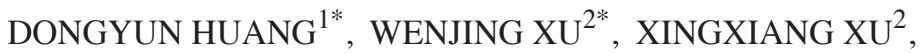 \\ XUDONG ZHANG ${ }^{1}$, RUI ZHOU ${ }^{3}$ and PING CHEN ${ }^{3}$ \\ Departments of ${ }^{1}$ Geriatric Medicine and ${ }^{2}$ Respiratory Medicine, Subei People's Hospital of Jiangsu Province, \\ Yangzhou, Jiangsu 225001; ${ }^{3}$ Department of Respiratory Medicine, The Second Xiangya Hospital \\ of Central South University, Changsha, Hunan 410011, P.R. China
}

Received February 23, 2016; Accepted August 3, 2016

DOI: $10.3892 / \mathrm{ol} .2016 .5315$

\begin{abstract}
Lung cancer is the most common cancer after breast and colon cancer, with high rates of mortality, worldwide. There are two main types of lung cancer, small cell lung carcinoma (SCLC), which accounts for approximately $20 \%$ of all lung cancer cases and non-SCLC, which accounts for almost $80 \%$ of lung cancer cases. Although lung cancer is one of the most aggressive types of cancer, progress in achieving better clinical outcomes has been gradual. Even though a number of markers have been suggested for the diagnosis of lung cancer and monitoring of disease progression, there is no clear way of assessing the invasion, epithelial-mesenchymal transition (EMT) and metastasizing capability of the primary tumor cells. We investigated the incidence of cytokeratin 19 (CK19)-negative expressers in different types of lung cancer from 111 lung cancer patients, their serum and pleural effusion CYFRA21-1 levels and whether induction of EMT in the primary focus cells influences the expression of CK19. In addition, we examined whether CK19-negative lung cancers were more invasive and metastatic. We also examined the propensity of primary focus cells to undergo EMT in the presence of transforming growth factor- $\beta 1$ (TGF- $\beta 1$ ). The results obtained suggested that the invasion and metastasis of lung tumor cells can be assessed by having a complete picture of serum CYFRA21-1 together with the CK19 expression status of primary focus cells and pleural effusion. This assessment may be further improved by examining the propensity of the isolated primary focus cells to undergo TGF- $\beta 1$ induced EMT in cell culture.
\end{abstract}

Correspondence to: Dr Wenjing Xu, Department of Respiratory Medicine, Subei People's Hospital, 98 West Nantong Road, Yangzhou, Jiangsu 225001, P.R. China

E-mail: wlxsmbka54@163.com

${ }^{*}$ Contributed equally

Key words: endothelial-mesenchymal transition, cytokeratin 19, squamous cell carcinoma, adenocarcinoma, small cell lung carcinoma, pleural effusion, CYFRA21-1, metastasis, transforming growth factor- $\beta 1$

\section{Introduction}

Lung cancer is the most common cancer after breast and colon cancer, worldwide (1). The incidence of lung cancer is similar to the mortality rates for this disease due to the high fatality of lung cancer. According to the data available from the International Agency for Research on Cancer, the annual lung cancer deaths are expected to increase to approximately 10 million by 2030 (2). The most common type of lung cancer histologically is adenocarcinoma, which accounts for almost $50 \%$ of all lung cancers (3).

Histologically there are two main types of lung cancer, small cell lung carcinoma (SCLC) and non-SCLC (NSCLC). SCLC accounts for approximatley $20 \%$ of all lung cancer cases, while NSCLC accounts for almost $80 \%$ of lung cancer cases (4). There are three histological subtypes of NSCLC: i) squamous cell carcinoma (SCC), ii) adenocarcinoma, and iii) large cell lung carcinoma, each accounting for 25 , 40 and $15 \%$ of the total NSCLC cases, respectively (5).

Despite the recognition of lung cancer as one of the most aggressive types of cancer, there is slow progress in the clinical outcomes even though a large number of new drugs are available. The most important issues for this drawback in the clinical handling of lung cancer is the unavailability of validated serum tumor markers, which are useful in both the diagnosis and prognosis of the disease (6). Many types of malignancy cause pleural effusions, and cancers that most frequently metastasize to the pleura are lung and breast carcinomas and lymphomas. Even though the cytological examination of pleural effusion is considered a standard approach for diagnosis, its sensitivity is typically only $50-70 \%(7,8)$.

A number of tumor markers, including carcinoembryonic antigen (CEA), carbohydrate antigen 125 (CA125), and CYFRA21-1, a fragment of cytokeratin 19 (CK19), have been evaluated as better and more accurate tumor markers in serum as well as pleural fluid in many studies $(7,8)$. It has also been observed that a combination of two or more markers is more powerful than a single marker. However, the real diagnosis predictability power of these markers was not assessed in many of these studies, since the cytological presence of tumor cells was detected in the pleural effusions of the patients (9-11). Many of the abovementioned markers were 
found to be elevated in the pleural effusions of cancer patients as compared to the benign pleural effusions (7). Pleural effusion-derived human lung cancer cells were found to be more invasive and metastatic than cancer cells from primary lesions and this difference may be related to epithelial-mesenchymal transition (EMT).

EMT, which usually plays an important role in embryonic tissue morphogenesis and in post-injury fibrosis $(12,13)$, is inappropriately reactivated during adulthood under certain pathological conditions such as cancer, and contributes to tumor metastasis (13). EMT is known to mediate the many alterations and the resultant phenotype modulation in tumor architecture. EMT is characterized by the disruption of intercellular adhesion, elevated tumor cell motility, decreased susceptibility to anoikis and apoptosis and in the release of cells from epithelial tissue (9-11). The released tumor cells, which are resistant to anoikis, assume mesenchymal-like phenotype that is suitable for migration, invasion and dissemination, all contributing to metastatic progression. In many cases, the degree of EMT of cancer cells determines the severity of cancer (14). EMT has also been demonstrated to be involved in resistance to anoikis, which is critical in the inhibition of cancer metastasis in various solid tumors (15).

Cytokeratins (CKs) are common tumor markers and are the main structural elements of the cytoskeleton in epithelial cells, and on the basis of structural properties CKs, there are 20 subtypes. Of these, CK19 is a soluble and acidic type I CK and is expressed in the epithelium lining of the bronchial tree and is known to be overexpressed in lung cancer tissues (16). There is elevated CK19 degradation in neoplastically transformed epithelial cells due to increased caspase-3 activity, and the proteolytic fragments, particularly, CYFRA21-1, are released into the blood. CK19 is considered to be closely associated with lung cancer; however, some studies reported CK19 expression to be negative in certain lung cancers (9-11). CK19 expression in some lung cancer cell lines was reduced following transforming growth factor (TGF)- $\beta$-induced EMT (17). Circulating levels of CK19 fragments including CYFRA21-1 likely reflect the extent of cytoskeletal formation in cancer cells and may also associate with the degree of tumor differentiation towards squamous epithelium (16).

In the present study, tumor samples from 111 lung cancer patients were employed, and the incidence of CK19-negative expressers in different types of lung cancer was investigated, as well as whether the induction of EMT in the primary focus cells influences the expression of CK19. We also examined whether CK19-negative lung cancers were more invasive and metastatic.

\section{Patients and methods}

General. All 111 patients were selected according to the 7th version TNM staging, described by the American Joint Committee on Cancer and Union for International Cancer Control in 2007 (9-11). Lung cancer patients diagnosed with different types of lung cancer at stage IV and treated with tyrosine kinase inhibitors or platinum $\left(75 \mathrm{mg} / \mathrm{m}^{2}\right)$ and docetaxel $\left(75 \mathrm{mg} / \mathrm{m}^{2}\right)$-based chemotherapy were recruited in the present study. The patients were admitted to the Subei People's Hospital between January, 2013 and December, 2014.
Table I. Patient characteristics.

\begin{tabular}{lcc}
\hline Characteristic & Males & Females \\
\hline $\begin{array}{l}\text { No. } \\
\text { Age (range, years) }\end{array}$ & 90 & 21 \\
Lung cancer stage & $44-82$ years & $40-75$ years \\
Histological type & 4 & 4 \\
Adenocarcinoma & & $14(1)$ \\
(CK19-negative cases) & $42(13)$ & $4(4)$ \\
$\begin{array}{l}\text { Small cell lung carcinoma } \\
\text { (CK19-negative cases) }\end{array}$ & $28(15)$ & $2(1)$ \\
$\begin{array}{l}\text { Squamous cell carcinoma } \\
\text { (CK19-negative cases) }\end{array}$ & $19(4)$ & $1(1)$ \\
$\begin{array}{l}\text { Adenosquamous } \\
\text { (CK19-negative cases) }\end{array}$ & $1(1)$ & \\
Treatment type & & 15 \\
Chemotherapy & & 5 \\
TKI & 77 & 1 \\
None & 5 & \\
\hline
\end{tabular}

CK19, cytokeratin 19; TKI, tyrosine kinase inhibitor.

The study was approved by the Ethics Committee of the Subei People's Hospital. Written informed consent was obtained from each participant. This study conforms to 'The Code of Ethics of the World Medical Association' (Declaration of Helsinki, 1964). The 111 patients comprised 90 men (44-82 years of age) and 21 women (40-75 years of age). Patient characteristics are shown in Table I. A total of 56 adenocarcinoma, 21 SCC, 32 SCLC and 2 adenosquamous carcinoma cases were identified. The enrolled patients matched the following criteria: Karnofsky score with a >60-year life expectancy of $>3$ months, no immune system diseases, normal electrocardiogram, normal liver and kidney function, and receiving no antitumor therapy within one month prior to the study. Tyrosine kinase inhibitor-treated cases were of the adenocarcinoma type.

Blood samples were collected from all the patients and sera were separated and frozen until subsequent analysis. Pleural effusion samples were collected by following the approved procedures and were examined cytologically and processed for immunohistochemistry.

Immunohistochemistry. Tumor tissue samples (primary foci) were processed for immunohistochemistry for the detection of CK19, E-cadherin and vimentin. Primary focus tissue samples were fixed in formalin and embedded in paraffin blocks. Multiple sections $(4 \mu \mathrm{m})$ from each sample were used for immunohistochemical analysis. The samples were stained with hematoxylin and eosin. Anti-CK19, anti-E-cadherin and anti-vimentin antibodies were purchased from TIYO Biotechnology Corporation (Shanghai, China). Anti-CK19 rabbit polyclonal antibody (catalog no. Z98123) was used at a dilution of 1:50; anti-E-cadherin CK19 rabbit polyclonal (catalog no. Z86603) antibody was used at a dilution of 1:100; and anti-vimentin CK19 rabbit polyclonal antibody (catalog 


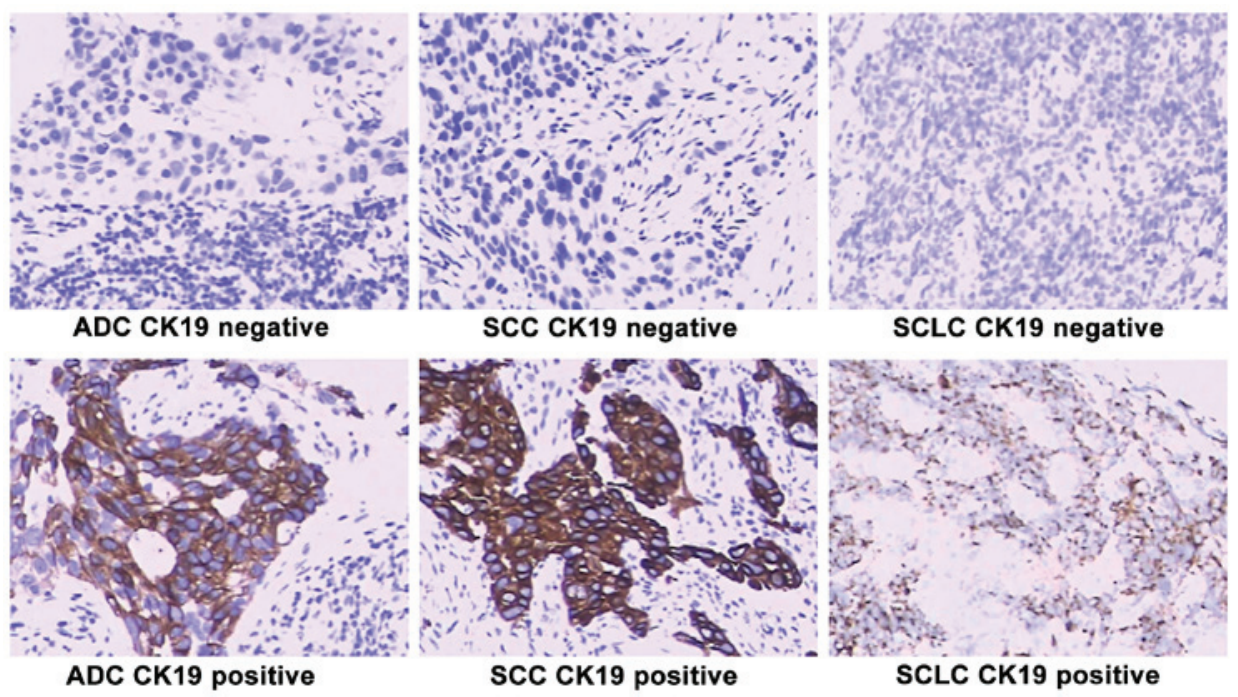

Figure 1. Immunohistochemical analysis of tumor marker cytokeratin 19 (CK19) expression in tumor samples. ADC, adenocarcinoma; SCC, squamous cell carcinoma; SCLC, small cell lung carcinoma.

no. Z40651) was used at a dilution of 1:500, followed by incubation with horseradish peroxidase-conjugated anti-IgG (Beijing CellChip Biotechnology Co., Ltd., Beijing, China) at a dilution of 1:100. Color development was performed using 3,3'-diaminobenzidine substrate.

Appearance of tan particles in the cell membrane and cytoplasm was considered positive for CK19, E-cadherin and vimentin, respectively. Immunohistochemical results were confirmed by two pathologists using a double-blinded method.

Marker analyses. A Roche E601 Cobas automatic chemiluminescence immunoassay analyzer (Roche Diagnostics, Basel, Switzerland) was used to measure serum CEA, CA125, neuron-specific enolase (NSE), $\alpha$-fetoprotein (AFP) and CYFRA21-1 in patient sera and pleural effusions. CYFRA21-1 $>3.3 \mathrm{ng} / \mathrm{ml}$, NSE $>15.2 \mathrm{ng} / \mathrm{ml}$, CEA $>5.0 \mathrm{ng} / \mathrm{ml}$, CA125 $>35.0 \mathrm{ng} / \mathrm{ml}$ and AFP $>7 \mathrm{ng} / \mathrm{ml}$ were considered positive values.

TGF- $\beta 1$-induced EMT in lung tumor cells. Tumor cells were isolated from primary focus tissues obtained from the patients. Primary cells were isolated from the primary tumor tissue and pleural effusion from the same patient and were cultured. The cells were isolated in two steps: i) centrifugation through lymphocyte separation medium (specific gravity of 1.077), followed by culturing in RPMI-1640 medium containing $20 \%$ fetal bovine serum (Beijing LabLead Biotechnology Corporation, Beijing, China) for 2 days. ii) Subsequently 40/20\% Percoll (Beijing Solarbio Science \& Technology Co., Ltd., Beijing, China) was used as second step centrifugation for separating the cells. The collected cells were cultured again in RPMI-1640 culture medium containing 20\% fetal bovine serum, and incubated at $37^{\circ} \mathrm{C}$ under $5 \% \mathrm{CO}_{2}$ atmosphere until 70-80\% confluency. The cells were treated with TGF- $\beta 1$ (purchased from Shanghai Kexing Biotech Co., Ltd., Shanghai, China) at $5 \mathrm{ng} / \mathrm{ml}$ for $24 \mathrm{~h}$. The cells were then washed and processed. Immunohistochemical analysis for E-cadherin, CK19 and vimentin, was performed prior to and after treatment with TGF- $\beta 1$, as described above. Presence or absence of these markers was scored as positive or negative, respectively.

Statistical analysis. $\mathrm{P}<0.05$ was considered statistically significant.

\section{Results}

Patient characteristics. The 111 patients comprised 90 men and 21 women, with a similar age distribution (40-80 years). The patients were diagnosed with stage IV lung cancer. Histologically, the majority of cases were adenocarcinoma type, followed by SCLC and SCC, both in the male and female patients (Table I). There were only two cases of adenosquamous carcinoma in this patient sample. The majority of the patients received chemotherapy, based on platinum- and docetaxel-based chemotherapy, while those with epidermal growth factor receptor gain-of-function mutations were administered tyrosine kinase inhibitor (TKI) therapy (Gefitinib). Only a small number of patients (5 men and 1 woman) did not receive these therapies due to financial constraints.

Histology of the primary tumor tissue. Tumor tissues from 111 patients were examined immunohistochemically for CK19 expression. While elevated CK19 expression was observed in certain lung cancers, a loss of CK19 is likely associated with EMT (17). In the present study, CK19 expression was absent in many SCLC tumors, $>50 \%$ in men and $100 \%$ in women. As such, CK19 expression was relatively decreased in SCLC tumors in comparison to other lung cancers (Fig. 1). Of the adenocarcinomas, approximately $31 \%$ of tumors from male patients were negative for CK19, which was much lower for women (7\%). Overall, SCLC tumor tissues showed more likelihood of EMT characteristics (Table I). There was an apparent reduction in the proportion of CK19-negative expresser primary tumors as well as pleural effusion cells following TKI therapy, in comparison to untreated or chemotherapy-treated patient tumors, in all the types of lung cancer (Fig. 2). 


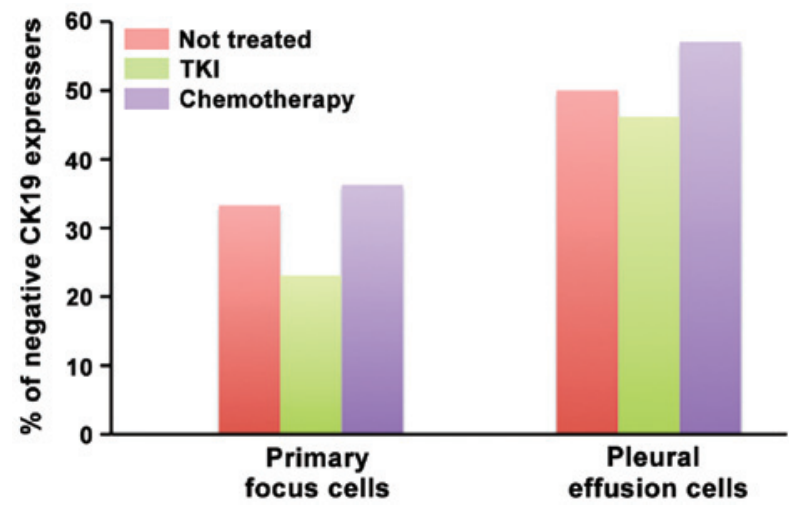

Figure 2. Percentage of tumors with cytokeratin 19 (CK19) negatives in lung cancer.

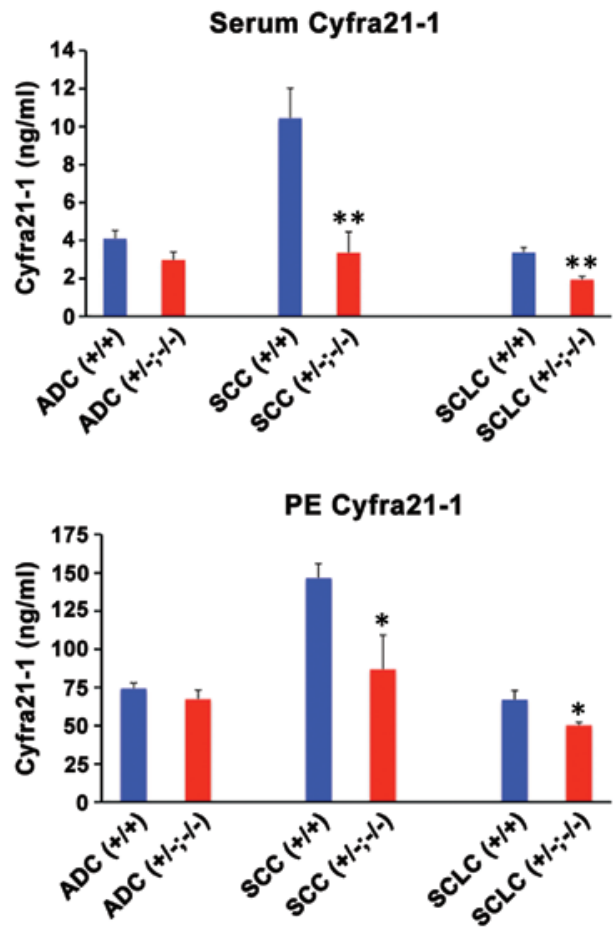

Figure 3. Assessment of CYFRA21-1 in serum and pleural effusion, taking into account the differential cytokeratin 19 (CK19) expression between primary focus and pleural effusion (PE) cells. CK19 expression in primary focus cells and in PE cells is indicated as +/+ or +/- or -/- in different types of lung cancer. ADC, adenocarcinoma; SCC, squamous cell carcinoma; SCLC, small cell lung carcinoma. ${ }^{*} \mathrm{P}<0.05$ vs. control and ${ }^{* *} \mathrm{P}<0.01$ vs. control.

CYFRA21-1 levels. Serum and pleural effusion CYFRA21-1 levels are considered important markers of malignancy for many types of cancer, in particular for lung cancer. Since CYFRA21-1 is a fragment of CK19, we examined the serum and pleural effusion CYFRA21-1 levels as a function of loss of CK19 expression in pleural effusion cells. The results showed that when CK19 was expressed strongly in the primary focus and pleural effusion cells, CYFRA21-1 levels were high and when CK19 expression was lost in the primary tumors and/or pleural effusion cells, CYFRA21-1 levels decreased significantly in all types of cancer (Fig. 3). Additionally, CYFRA21-1 levels were much higher in the serum and pleural effusion of patients with CK19-positive SCC tumors in comparison to

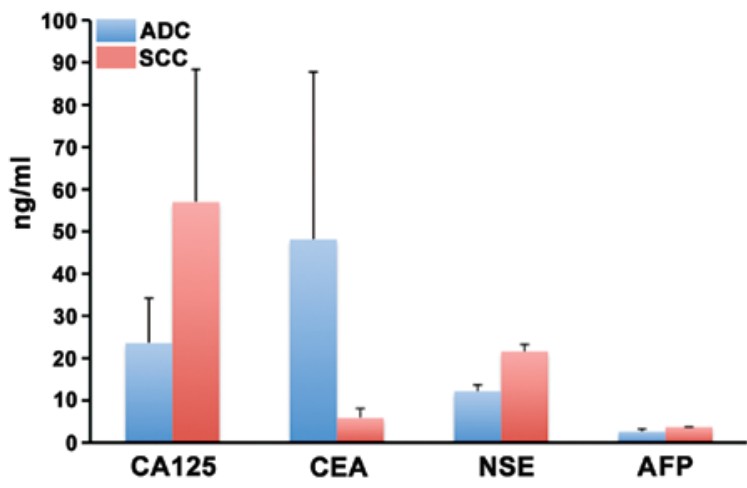

Figure 4. Serum tumor markers in lung cancer patients. ADC, adenocarcinoma; SCC, squamous cell carcinoma; CA125, carbohydrate antigen 125 ; CEA, carcinoembryonic antigen; NSE, neuron-specific enolase; AFP, $\alpha$-fetoprotein.

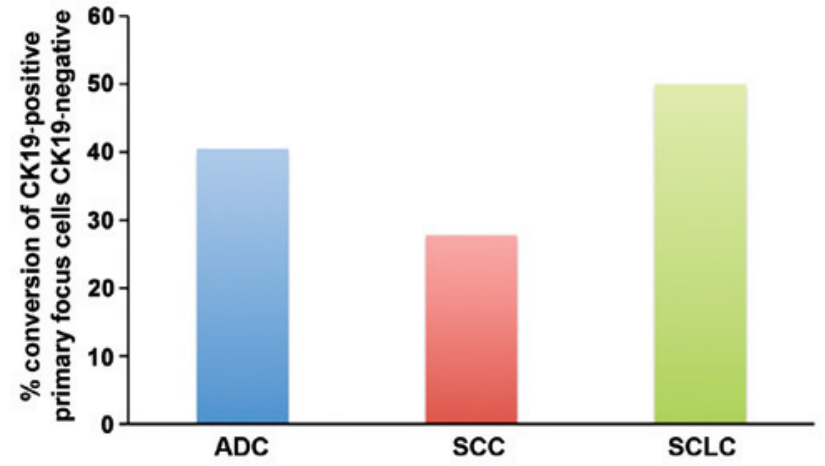

Figure 5. EMT induction in primary focus cells by transforming growth factor- $\beta 1$ : Conversion of cytokeratin 19 (CK19) positive cells of primary focus to CK19 negative cells. ADC, adenocarcinoma; SCC, squamous cell carcinoma; SCLC, small cell lung carcinoma.

other types of tumors (Fig. 2). The high levels of CYFRA21-1 in SCC patient sera and pleural effusion likely reflects the strong presence of CK19 in positive tumors (Fig. 1). The results also suggested that serum CYFRA21-1 levels were elevated only marginally in the lung cancers as compared to the benign base levels $(1.3-2.6 \mathrm{ng} / \mathrm{ml})$ with the exception of SCC, where the increase was much above the normal range (Fig. 3). However, CYFRA21-1 levels were greatly elevated above the benign range $(6.5-35 \mathrm{ng} / \mathrm{ml})(18)$ in pleural effusion of the lung cancer patients, suggesting that the pleural effusion measurement of CYFRA21-1 is an optimal tumor marker.

Other serum markers. Markers of tumor malignancy including CA125, CEA, NSE and AFP were measured in the sera of a few of the patients and there was considerable variation among the patients and the levels. Thus, for patients with ADC, the average of CA125, CEA, NSE and AFP was 23.6 $\pm 10.9,48.3 \pm 39.7$, $10.1 \pm 2.4$, and $2.1 \pm 0.6$, respectively. For patients with SCC, the average of CA125, CEA, NSE and AFP was 57.1 $\pm 31.5,5.6 \pm 2.7$, $20.8 \pm 2.5$, and 2.6 \pm 0.4 respectively None of the markers was significantly different from the normal range (Fig. 4).

Induction of EMT in primary focus cells by TGF- $\beta 1$. TGF- $\beta 1$ when incubated with primary focus lung tumor cells created 

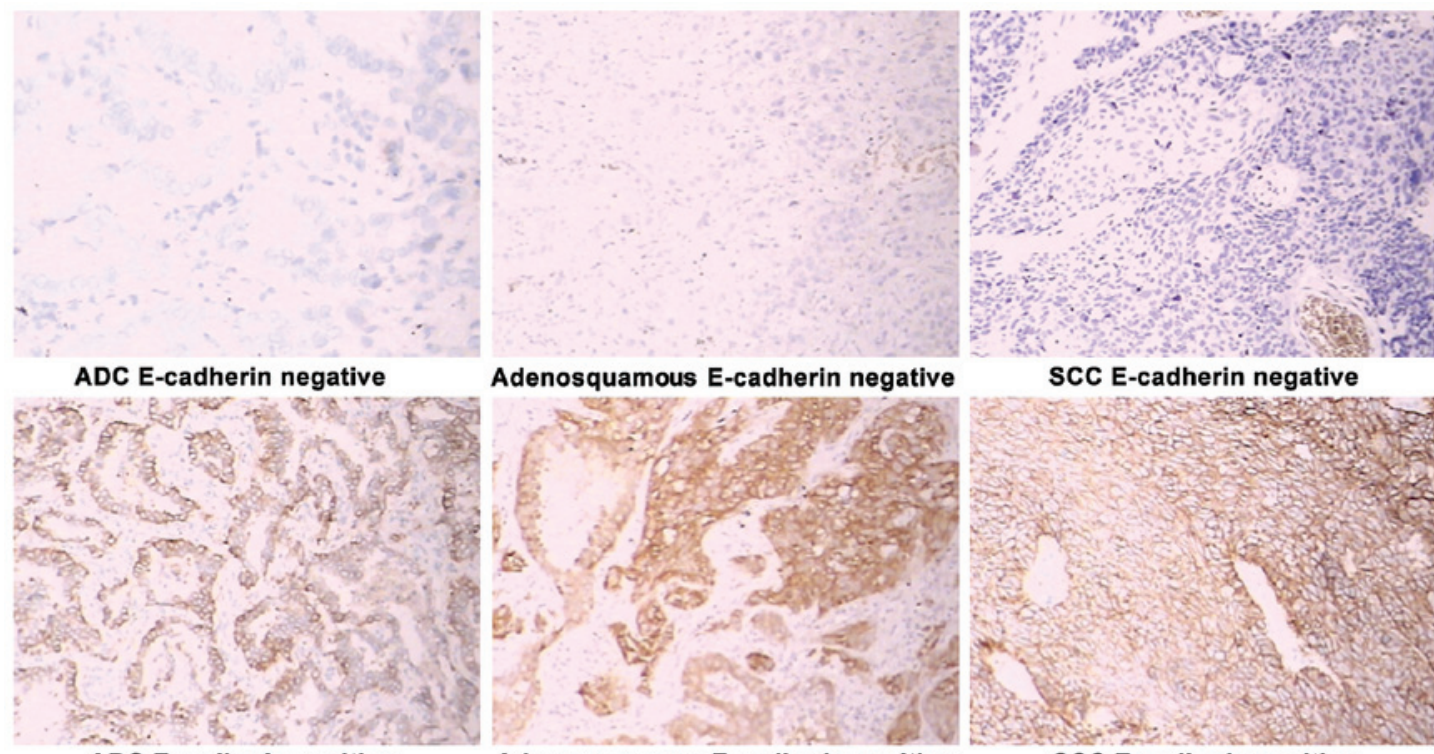

SCC E-cadherin negative

ADC E-cadherin positive

Adenosquamous E-cadherin positive

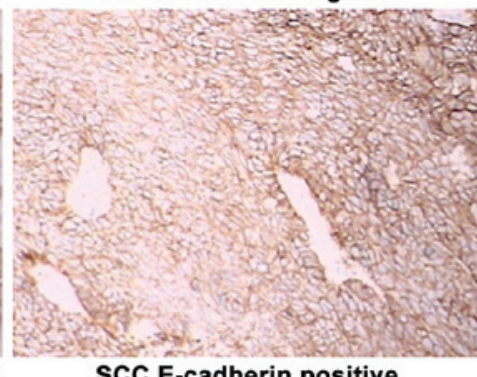

SCC E-cadherin positive

Figure 6. Immunohistochemical analysis of tumor marker E-cadherin expression in tumor samples. ADC, adenocarcinoma; SCC, squamous cell carcinoma.

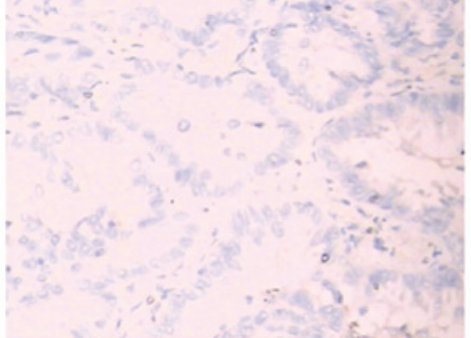

ADC vimentin negative

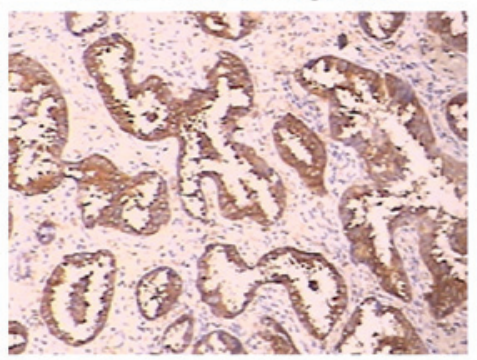

ADC vimentin positive

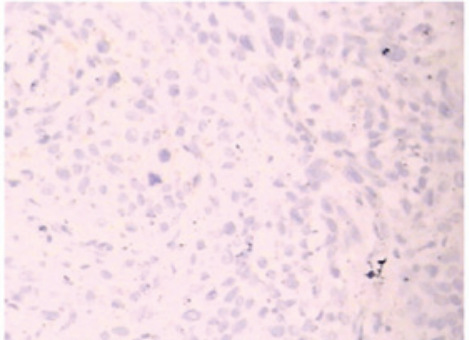

SCC vimentin negative

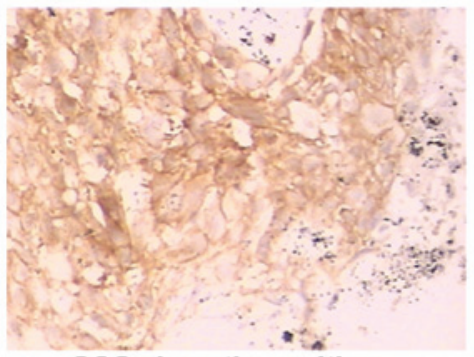

SCC vimentin positive

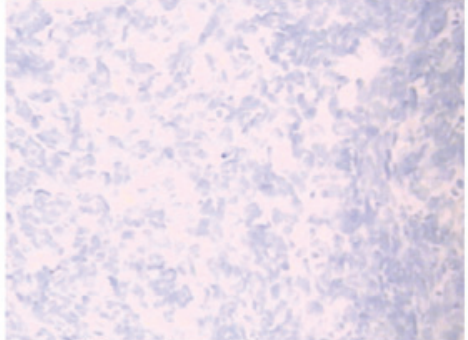

SCLC vimentin negative

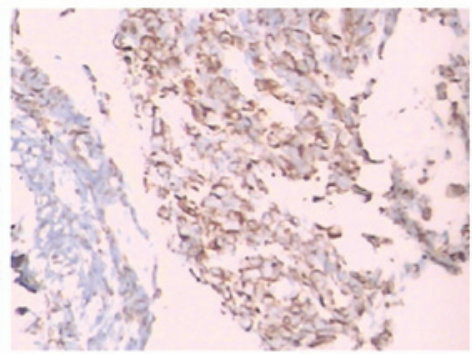

SCLC vimentin positive

Figure 7. Immunohistochemical analysis of tumor marker vimentin expression in tumor samples. ADC, adenocarcinoma; SCC, squamous cell carcinoma; SCLC, small cell lung carcinoma.

changes relevant for EMT, in many cases. Thus, in almost $40 \%$ of adenocarcinoma tumors, CK19-positive primary focus cells were induced to lose CK19 expression and undergo EMT by TGF- $\beta 1$. This proportion of tumors was $28 \%$ for SCC and $50 \%$ for SCLC (Fig. 5). Thus, SCLC primary focus cells are inducible to undergo EMT by TGF- $\beta 1$ more readily. As mentioned above, SCLC primary cells show a higher level of EMT even without induction by TGF- $\beta 1$ (Table I).

Another set of important markers of EMT is loss of E-cadherin and gain of vimentin expression. E-cadherin was found to be negative in almost $37.5 \%$ of adenocarcinoma primary tumor cells, $52.3 \%$ of SCC tumor cells and $22 \%$ of SCLC tumor cells (Fig. 6), prior to treating with TGF- $\beta 1$. By contrast, vimentin expression in these tumors was positive in

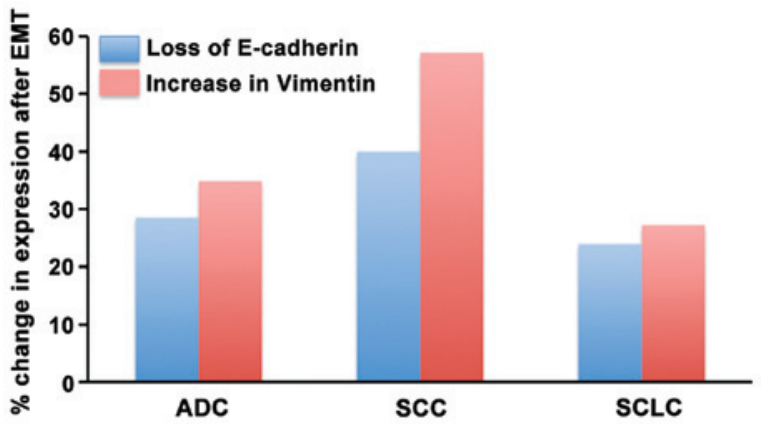

Figure 8. Transforming growth factor- $\beta$-induced epithelial-mesenchymal transition (EMT) in primary focus cells of lung cancers causes loss of E-cadherin and gain of vimentin expression. ADC, adenocarcinoma; SCC, squamous cell carcinoma; SCLC, small cell lung carcinoma. 
$23.2 \%$ of adenocarcinoma tumor cells, $33.3 \%$ of SCC tumor cells and $31.3 \%$ SCLC tumor cells (Fig. 7). Following treatment of lung tumor primary focus cells with TGF- $\beta 1$, the loss of E-cadherin expression was always associated with an increase in vimentin expression (Fig. 8). The percentage of SCC tumors that showed this response to TGF- $\beta 1$ ( $40 \%$ change in E-cadherin expression and $57 \%$ in vimentin expression) was higher than that for adenocarcinoma and SCLC (Fig. 6).

\section{Discussion}

Comparative expression status of CK19 in primary lung cancer tissues and in pleural effusions can be useful in assessing the metastasizing ability of these cancer cells. Thus, if a patient's pleural effusion cells have a higher level of negative expression of CK19 when compared with the same patient's primary lung cancer tissues, it is assumed that the negative CK19 expression is associated with invasion and metastasis (19). Thus, in the present study, a large proportion of patients with SCLC tumors had tumor tissue with no expression of CK19 and the pleural effusion cells in these patients showed a lack of CK19 expression, indicating a high degree of metastasis in this type of lung cancer, which is a typical characteristic of SCLC (20). Several reasons for the loss of CK19 expression have been suggested including altered expression and enhanced proteolytic degradation (21).

Since CYFRA21-1 is a fragment of CK19, serum and pleural effusion CYFRA21-1 levels are considered important markers of malignancy for many types of cancer, in particular for lung cancer $(7,21)$. However, it is difficult to be certain regarding cancer diagnosis based on circulating CYFRA21-1 levels as there are lung cancer cases where there are decreased CYFRA21-1 levels while there is an increase in other cases $(7,21)$. However, from the present findings, serum CYFRA21-1 levels decreased with the increased possibility of EMT, as detected by loss of CK19 expression in pleural effusions in comparison to the corresponding primary tumor tissue. Thus, serum CYFRA21-1 levels along with CK19 expression status of cancer cells from primary focus and pleural effusions can positively identify the invasion and metastasis ability of the lung cancer cells. Other markers including CA125, CEA, AFP and NSE, which are commonly used for cancer diagnosis, were not consistent in this assessment.

The metastasizing ability is bestowed upon the primary cancer cells via EMT and one of the primary factors that can induce EMT is TGF- $\beta 1$. Although TGF- $\beta 1$ normally functions as an inhibitor of epithelial cell proliferation, because of altered signaling pathways in many cancer cells, TGF- $\beta 1$ acts to enhance the proliferation of cancer cells, including lung cancer cells (22). In addition to enhancing cancer cell growth, TGF- $\beta 1$ is known to promote EMT in cancer cells and thus contribute to cancer cell invasion and metastasis. It has been suggested that in the early stages of primary tumor development in epithelial tissues, TGF- $\beta 1$ acts as an inhibitor of tumor growth via cell cycle arrest and apoptosis $(23,24)$. However, as tumor progression occurs, during the later stages of tumor development, there is either inactivation of the TGF- $\beta 1$ signaling pathway or aberrant regulation of the cell cycle and the cancer cells become resistant to growth inhibition by TGF- $\beta 1(23,24)$. Under these conditions TGF- $\beta 1$ is used as a growth promoter by the cancer cells (25). The expression of mRNA and protein of TGF- $\beta 1$ was greatly elevated in many types of cancer, including those of the pancreas, colon, stomach, lung, endometrium, prostate, breast, brain, and bone (26).

Considering that the propensity to undergo EMT is enhanced in the presence of a cytokine such as TGF- $\beta 1$, which is known to be elevated in lung cancer patients, we examined the EMT inducibility of primary focus cells, ex vivo, by TGF- $\beta 1$. Depending on the EMT marker employed, SCLC and SCC tumors had a higher ability to undergo TGF- $\beta 1$-induced EMT. SCLC primary focus cells are inducible to undergo EMT by TGF- $\beta 1$ more readily, if we consider loss of CK19 expression. There is in fact, a higher degree of EMT, in terms of lack of CK19 in SCLC primary tumor cells. By contrast, SCC tumor primary cells respond by the loss of E-cadherin and elevated vimentin expressions. Downregulation of E-cadherin, together with the upregulation of N-cadherin characterizes the EMT process and these changes in the expression of these proteins is associated with the acquisition of resistance to apoptosis and anoikis $(27,28)$.

In summary, the results of the present study suggest that the invasion and metastasis of lung tumor cells can be assessed by having a complete picture of serum CYFRA21-1 together with the CK19 expression status of primary focus cells and pleural effusion. This assessment may be further improved by examining the propensity of the isolated primary focus cells to undergo TGF- $\beta 1$-induced EMT in cell culture.

\section{Acknowledgements}

The authors would like to acknowledge technical assistance from Dr Yong Chen and Dr Jixin Jiang.

\section{References}

1. Jemal A, Bray F, Center MM, Ferlay J, Ward E and Forman D: Global cancer statistics. CA Cancer J Clin 61: 69-90, 2011.

2. Haghgoo SM, Allameh A, Mortaz E, Garssen J, Folkerts G, Barnes PJ and Adcock IM: Pharmacogenomics and targeted therapy of cancer: Focusing on non-small cell lung cancer. Eur J Pharmacol 754: 82-91, 2015.

3. Morales-Oyarvide V and Mino-Kenudson M: High-grade lung adenocarcinomas with micropapillary and/or solid patterns: A review. Curr Opin Pulm Med 20: 317-323, 2014.

4. Burdett S, Stewart LA and Rydzewska L: A systematic review and meta-analysis of the literature: Chemotherapy and surgery versus surgery alone in non-small cell lung cancer. J Thorac Oncol 1: 611-621, 2006.

5. Travis WD: Pathology of lung cancer. Clin Chest Med 23: 65-81, viii, 2002.

6. Bastawisy AE, Azzouny ME, Mohammed G, Allah AA and Behiry E: Serum cytokeratin 19 fragment in advanced lung cancer: Could we eventually have a serum tumor marker? Ecancermedicalscience 8: 394, 2014.

7. Hsieh TC, Huang WW, Lai CL, Tsao SM and Su CC: Diagnostic value of tumor markers in lung adenocarcinoma-associated cytologically negative pleural effusions. Cancer Cytopathol 121: 483-488, 2013.

8. Liang QL, Shi HZ, Qin XJ, Liang XD, Jiang J and Yang HB: Diagnostic accuracy of tumour markers for malignant pleural effusion: A meta-analysis. Thorax 63: 35-41, 2008.

9. Miedouge M, Rouzaud P, Salama G, et al: Evaluation of seven tumour markers in pleural fluid for the diagnosis of malignant effusions. Br J Cancer 81: 1059-1065, 1999.

10. Huang WW, Tsao SM, Lai CL, Su CC and Tseng CE: Diagnostic value of Her-2/neu, Cyfra 21-1, and carcinoembryonic antigen levels in malignant pleural effusions of lung adenocarcinoma. Pathology 42: 224-228, 2010. 
11. Riantawan P, Sangsayan P, Bangpattanasiri K and Rojanaraweewong P: Limited additive value of pleural fluid carcinoembryonic antigen level in malignant pleural effusion. Respiration 67: 24-29, 2000.

12. Hay ED: The mesenchymal cell, its role in the embryo, and the remarkable signaling mechanisms that create it. Dev Dyn 233: 706-720, 2005.

13. Guarino M, Micheli P, Pallotti F and Giordano F: Pathological relevance of epithelial and mesenchymal phenotype plasticity. Pathol Res Pract 195: 379-389, 1999.

14. Guarino M,Rubino B and Ballabio G: The role of epithelial-mesenchymal transition in cancer pathology. Pathology 39: 305-318, 2007.

15. Chunhacha P, Sriuranpong V and Chanvorachote P: Epithelialmesenchymal transition mediates anoikis resistance and enhances invasion in pleural effusion-derived human lung cancer cells. Oncol Lett 5: 1043-1047, 2013.

16. Kosacka M and Jankowska R: Comparison of cytokeratin 19 expression in tumor tissue and serum CYFRA 21-1 levels in non-small cell lung cancer. Pol Arch Med Wewn 119: 33-37, 2009.

17. Xu GP, Li QQ, Cao XX, Chen Q, Zhao ZH, Diao ZQ and Xu ZD: The effect of TGF- $\beta 1$ and Smad7 gene transfer on the phenotypic changes of rat alveolar epithelial cells. Cell Mol Biol Lett 12: 457-472, 2007.

18. Romero S, Fernández C, Arriero JM, Espasa A, Candela A Martín C and Sánchez-Payá J: CEA, CA 15-3 and CYFRA 21-1 in serum and pleural fluid of patients with pleural effusions. Eur Respir J 9: 17-23, 1996.
19. Fujisue M, Nishimura R, Okumura Y, Tashima R, Nishiyama $Y$, Osako T, Toyozumi Y and Arima N: Clinical significance of ck19 negative breast cancer. Cancers (Basel) 5: 1-11, 2012.

20. Pietanza MC, Byers LA, Minna JD and Rudin CM: Small cell lung cancer: Will recent progress lead to improved outcomes? Clin Cancer Res 21: 2244-2255, 2015.

21. Ono A, Takahashi T, Mori K, Akamatsu H, Shukuya T, Taira T, Kenmotsu H, Naito T, Murakami H, Nakajima T, et al: Prognostic impact of serum CYFRA 21-1 in patients with advanced lung adenocarcinoma: A retrospective study. BMC Cancer 13: 354, 2013.

22. Bachman KE and Park BH: Duel nature of TGF-beta signaling: Tumor suppressor vs. tumor promoter. Curr Opin Oncol 17: 49-54, 2005.

23. Wakefield LM and Roberts AB: TGF- $\beta$ signaling: positive and negative effects on tumorigenesis. Curr Opin Genet Dev 12: 22-29, 2002.

24. Akhurst RJ and Derynck R: TGF- $\beta$ signaling in cancer - a double-edged sword. Trends Cell Biol 11: S44-S51, 2001.

25. Jakowlew SB: Transforming growth factor-beta in cancer and metastasis. Cancer Metastasis Rev 25: 435-457, 2006.

26. Gold LI: The role for transforming growth factor-beta (TGF-beta) in human cancer. Crit Rev Oncog 10: 303-360, 1999.

27. Guadamillas MC, Cerezo A and Del Pozo MA: Overcoming anoikis--pathways to anchorage-independent growth in cancer. J Cell Sci 124: 3189-3197, 2011.

28. Ko H, Kim S, Jin CH, Lee E, Ham S, Yook JI and Kim K: Protein kinase casein kinase 2-mediated upregulation of $\mathrm{N}$-cadherin confers anoikis resistance on esophageal carcinoma cells. Mol Cancer Res 10: 1032-1038, 2012. 